\title{
Beam Test Results of 3D Pixel Detectors Constructed With poly-crystalline CVD diamonds
}

\section{The RD42 Collaboration}

M. Reichmann ${ }^{* 24}$, A. Alexopoulos ${ }^{3}$, M. Artuso ${ }^{20}$, F. Bachmair ${ }^{24}$, L. Bäni ${ }^{24}$, M. Bartosik ${ }^{3}$, H. Beck ${ }^{23}$, V. Bellini' ${ }^{2}$, V. Belyaev ${ }^{12}$, B. Bentele ${ }^{19}$, A. Bes ${ }^{27}$, J-M. Brom ${ }^{7}$, M. Bruzzi ${ }^{4}$, G. Chiodini' ${ }^{26}$, D. Chren ${ }^{18}$, V. Cindro ${ }^{9}$, G. Claus ${ }^{7}$, J. Collot $^{27}$, J. Cumalat ${ }^{19}$, A. Dabrowski ${ }^{3}$, R. D'Alessandro ${ }^{4}$, D. Dauvergne ${ }^{27}$, W. de Boer ${ }^{10}$, C. Dorfer $^{24}$, M. Dünser ${ }^{3}$, G. Eigen ${ }^{30}$, V. Eremin ${ }^{6}$, G. Forcolin ${ }^{22}$, J. Forneris ${ }^{15}$, L. Gallin-Martel ${ }^{27}$, M-L. Gallin-Martel' ${ }^{27}$, K. K. Gan ${ }^{13}$, M. Gastal ${ }^{3}$, M. Goffe ${ }^{7}$, J. Goldstein ${ }^{17}$, A. Golubev ${ }^{8}$, A. Gorišek ${ }^{9}$, E. Grigoriev ${ }^{8}$, J. Grosse-Knetter ${ }^{23}$, A. Grummer ${ }^{21}$, M. Guthoff ${ }^{3}$, B. Hiti ${ }^{9}$, D. Hits ${ }^{24}$, M. Hoeferkamp ${ }^{21}$, T. Hofmann ${ }^{3}$, J. Hosselet ${ }^{7}$, F. Hügging ${ }^{1}$, C. Hutton ${ }^{17}$, J. Janssen ${ }^{1}$, H. Kagan ${ }^{13}$, K. Kanxheri ${ }^{28}$, R. Kass ${ }^{13}$, M. Kis ${ }^{5}$, G. Kramberger ${ }^{9}$, S. Kuleshov ${ }^{8}$, A. Lacoste ${ }^{27}$, S. Lagomarsino ${ }^{4}$, A. Lo Giudice ${ }^{15}$, I. Lopez Paz ${ }^{22}$, E. Lukosi ${ }^{25}$, C. Maazouzi ${ }^{7}$, I. Mandić ${ }^{9}$, C. Mathieu ${ }^{7}$, M. Menichelli ${ }^{28}$, M. Mikuž ${ }^{9}$, A. Morozzi ${ }^{28}$, J. Moss ${ }^{29}$, R. Mountain ${ }^{20}$, A. Oh $^{22}$, P. Olivero $^{15}$, D. Passeri ${ }^{28}$, H. Pernegger ${ }^{3}$, R. Perrino ${ }^{26}$, F. Picollo ${ }^{15}$, M. Pomorski ${ }^{11}$, R. Potenza ${ }^{2}$, A. Quadt ${ }^{23}$, F. Rarbi ${ }^{27}$, A. Re ${ }^{15}$, S. Roe ${ }^{3}$, D. A. Sanz Becerra ${ }^{24}$, M. Scaringella ${ }^{4}$, C. J. Schmidt ${ }^{5}$, S. Schnetzer ${ }^{14}$, E. Schioppa ${ }^{3}$, S. Sciortino ${ }^{4}$, A. Scorzoni ${ }^{28}$, S. Seidel ${ }^{21}$, L. Servoli ${ }^{28}$, D. S. Smith ${ }^{13}$, B. Sopko ${ }^{18}$, V. Sopko ${ }^{18}$, S. Spagnolo ${ }^{26}$, S. Spanier ${ }^{25}$, K. Stenson ${ }^{19}$, R. Stone ${ }^{14}$, B. Stugu ${ }^{30}$, C. Sutera ${ }^{2}$, M. Traeger $^{5}$, W. Trischuk ${ }^{16}$, M. Truccato ${ }^{15}$, C. Tuve $^{2}$, J. Velthuis ${ }^{17}$, N. Venturi ${ }^{3}$, S. Wagner $^{19}$, R. Wallny ${ }^{24}$, J. C. Wang ${ }^{20}$, N. Wermes ${ }^{1}$, M. Yamouni ${ }^{27}$, J. Zalieckas ${ }^{30}$ and M. Zavrtanik ${ }^{9}$

${ }^{1}$ Universität Bonn, Germany, ${ }^{2}$ INFN/University of Catania, Italy, ${ }^{3}$ CERN, Switzerland, ${ }^{4}$ INFN/University of Florence, Italy, ${ }^{5}$ GSI, Germany, ${ }^{6}$ Ioffe Institute, Russia, ${ }^{7}$ IPHC, France, ${ }^{8}$ ITEP, Russia, ${ }^{9}$ Jožef Stefan Institute, Slovenia, ${ }^{10}$ Universität Karlsruhe, Germany, ${ }^{11}$ CEA-LIST Technologies Avancées, France, ${ }^{12}$ MEPHI Institute, Russia, ${ }^{13}$ The Ohio State University, USA, ${ }^{14}$ Rutgers University, USA, ${ }^{15}$ University of Torino, Italy, ${ }^{16}$ University of Toronto, Canada, ${ }^{17}$ University of Bristol, UK, ${ }^{18}$ Czech Technical University, Czech Republic, ${ }^{19}$ University of Colorado, USA, ${ }^{20}$ Syracuse University, USA, ${ }^{21}$ University of New Mexico, USA, ${ }^{22}$ University of Manchester, UK, ${ }^{23}$ Universität Göttingen, Germany, ${ }^{24}$ ETH Zürich, Switzerland, ${ }^{25}$ University of Tennessee, USA, ${ }^{26}$ INFN-Lecce, Italy, ${ }^{27}$ LPSC-Grenoble, France, ${ }^{28}$ INFN-Perugia, Italy, ${ }^{29}$ California State University, USA, ${ }^{30}$ University of Bergen, Norway E-mail:

As a possible candidate for extremely radiation tolerant tracking devices we present a novel detector design - namely 3D detectors - based on poly-crystalline CVD diamond sensors with a pixel readout. The fabrication of recent 3D detectors as well their results in recent beam tests are presented. We measured the hit efficiency and signal response of two 3D diamond detectors with $50 \times 50 \mu \mathrm{m}$ cell sizes using pixel readout chip technologies currently used at CMS and ATLAS. In all runs, both devices attained efficiencies $>98 \%$ in a normal incident test beam of minimum ionising particles. The highest efficiency observed during the beam tests was $99.2 \%$.

29th International Symposium on Lepton Photon Interactions at High Energies

5-10 August 2019

Westin Harbour Castle, Toronto Canada 


\section{Introduction}

The radiation levels of the High-Luminosity-LHC (HL-LHC) are expected to be a big challenge for the future detectors. By 2028 experiments must be prepared for an instantaneous luminosity of $7.5 \cdot 10^{34} \mathrm{~cm}^{-2} \mathrm{~s}^{-1}$. In this environment the innermost tracking layer at a transverse distance of $\sim 30 \mathrm{~mm}$ to the interaction point will be exposed to a total fluence of $2 \cdot 10^{16} \mathrm{n}_{\mathrm{eq}} / \mathrm{cm}^{2}$ [四]. The expected lifetime of the current planar silicon tracking detectors would be about one year in such an environment.

In this work, we present the test beam measurements of 3D detectors using poly-crystalline Chemical Vapour Deposition (pCVD) diamond, which were fabricated by the CERN RD42 collaboration [ []]. Diamond has an average threshold displacement energy of $43 \mathrm{eV} /$ atom [B] which makes it intrinsically radiation tolerant and its band gap of $5.5 \mathrm{eV}$ [䧃] greatly simplifies the construction of the detectors as well as guarantees negligible leakage currents.

After the doses expected in the HL-LHC, all detector materials will be trap limited with a schubweg, the average drift distance before a free charge carrier gets trapped, below $75 \mu \mathrm{m}$ [ [1, 团]. The RD42 collaboration is studying a novel detector design in diamond, namely 3D detectors, to extend the radiation tolerance of diamond to fluences exceeding the HL-LHC doses. This detector design places column-like electrodes inside the detector material. In this detector geometry the drift distance an electron-hole pair must undergo to reach an electrode can be reduced below the schubweg of an irradiated sensor without reducing the number of electron-hole pairs created. The details of the general working principles of 3D detectors may be found in [ $[$ ].

\section{3D Pixel Detectors}

\subsection{Fabrication}

All devices discussed in this article were constructed with pCVD diamond. The sensors were thin plates with a thickness of $\sim 500 \mu \mathrm{m}$ and a side length of $\sim 5 \mathrm{~mm}$. In order to manufacture the electrodes in diamond, columns were fabricated perpendicular to the large side using a $130 \mathrm{fs}$ laser with a wavelength of $800 \mathrm{~nm}$. After focusing to a $2 \mu \mathrm{m}$ spot the laser has the energy density to convert diamond into a electrically resistive mixture of different carbon phases [ $[8]$. A Spatial Light Modulator (SLM) [9] was used to correct spherical aberrations during fabrication. This helped to achieve the high column yield of $\gtrsim 99.8 \%$, a column diameter of $2.6 \mu \mathrm{m}$ and a resistivity of the columns of the order of $0.1 \sim 1 \Omega \mathrm{cm}$. The yield was measured by manually counting missing or broken columns and the diameter was optically measured. The columns are not drilled completely through the diamond, but with a gap of $15 \mu \mathrm{m}$ to the opposite surface to avoid high voltage breakthrough at the operated bias voltages of up to $70 \mathrm{~V}$. The detector was constructed by ganging all bias columns together with a mesh metallisation on one surface and metallising the other surface to connect a small number of readout columns in order to match the pitch of the readout electronics for bump bonding.

The detectors described herein were connected to two different readout chips (ROCs), which is why different bonding processes, as shown in Figures $\mathrm{W}$, were used. For both of these detectors

\footnotetext{
* Speaker.
} 
a 3D cell size of $50 \mu \mathrm{m} \times 50 \mu \mathrm{m}$ was chosen. Since the layout of the ROCs had a different pixel pitch several cells were ganged together by connecting several readout columns with the surface metallisation. Detector 1 was connected to the PSI46digV2.1respin ROC [ए]] with a $3 \times 2$ cell ganging to match the pixel pitch of $150 \mu \mathrm{m} \times 100 \mu \mathrm{m}$. It was bump bonded to the ROC at the Nanofabrication Lab at the Princeton University [U]] with indium. This was achieved by putting equal height indium columns on both ROC and the sensor and then pressing them together without reflow (see Figure [a). The second detector was connected to the FE-I4B ROC [ए2] with a $5 \times 1$ cell ganging due to the pixel pitch of $250 \mu \mathrm{m} \times 50 \mu \mathrm{m}$. The bump bonding for this sensor was performed at IFAE-CNM in Barcelona [[13], 니] by an adapted process with tin-silver bumps (see Figure [b).

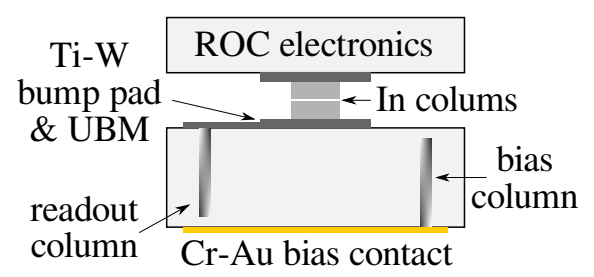

(a) CMS PSI46dig

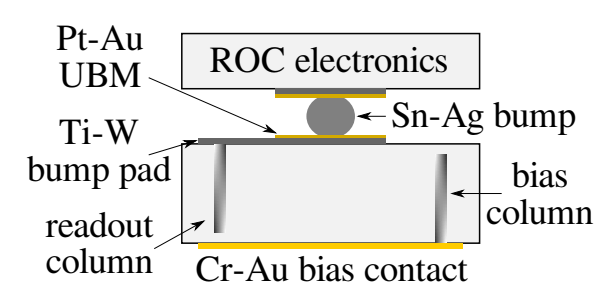

(b) ATLAS FE-I4B

Figure 1: Bump Bonding and metallisation for two different ROCs.

Both devices have $\sim 3500$ 3D cells, where one cell consists of four bias electrodes and one readout electrode in the centre. Since the bias electrodes are shared between the cells, $\sim 7200$ columns had to be drilled to build such a device. Except for the different readout, the sensors are very similar. Photographs of the assembled 3D detectors on the ROCs are shown in Figure [.].

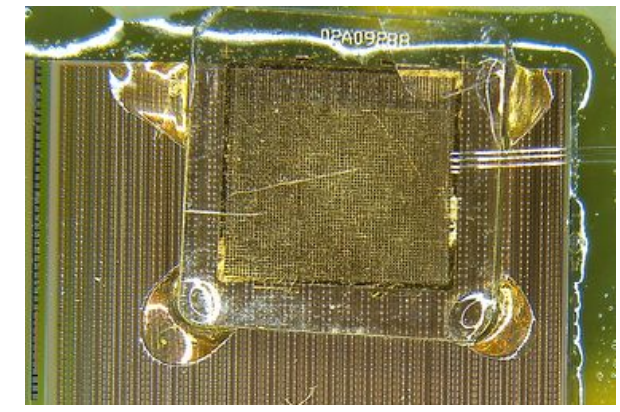

(a) Detector 1 with $3 \times 2$ ganging.

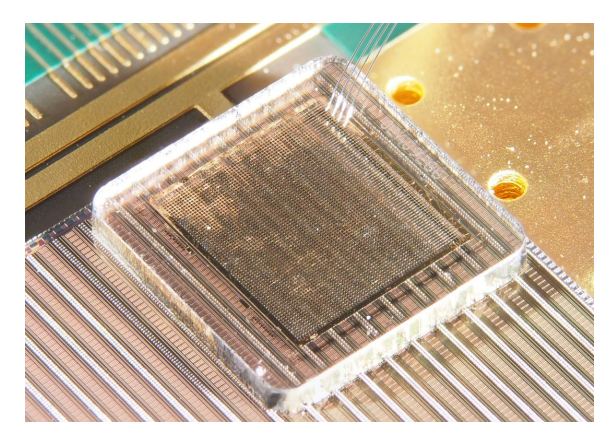

(b) Detector 2 with $5 \times 1$ ganging.

Figure 2: Assembled 3D pixel detectors.

\subsection{Results of Detector 1 ( $3 \times 2$ cell ganging)}

This detector was tested with pixel telescopes in beam lines at Paul Scherrer Institut (PSI) and the SPS facility at CERN in order to get both measurements at high rates as well as with high tracking resolution. At PSI a beam of $210 \mathrm{MeV} / \mathrm{c}$ positive pions was used [प5], where protons were removed with a plastic absorber. Whereas at CERN the momentum of the positive hadron beam was $120 \mathrm{GeV} / \mathrm{c}[\mathbb{1 0}]$. The preliminary beam test results show that, relative to a planar silicon device, the 
efficiency within a selected fiducial area was $99.2 \%$. Where the hit efficiency was defined as the percentage of hits in the 3D pixel detector when a particle track traversed the detector. This value was measured at both beam test facilities. The fiducial area was selected such as to exclude nonworking 3D cells found by visual inspection, which can happen due to broken or missing columns or due to metallisation issues, where the metal is not properly connected to the electrodes. A small discrepancy between a 3D and a fully efficient planar device is partly expected due to the relative inefficiency of the columns themselves. The area of the 3D columns compared to the whole cell is $0.4 \%$.

Figure [ $]$ shows that the efficiency of the detector plateaus at a voltage of $30 \mathrm{~V}$. This demonstrates that the device already operates well at very low voltages compared to a planar diamond detector. The efficiency at both facilities agrees well, which shows that the detector is independent of the different particle energies and intensities which were used.

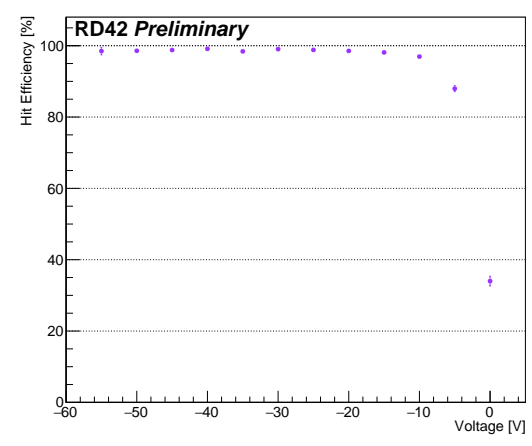

(a) PSI.

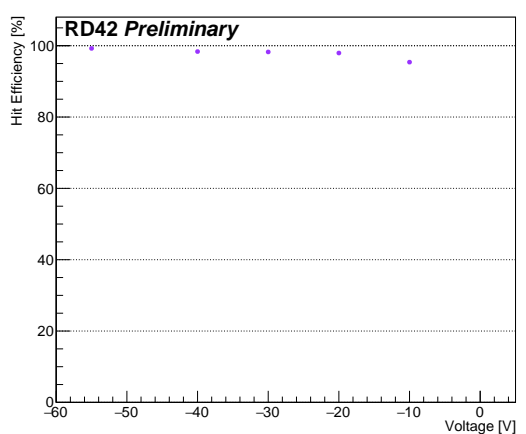

(b) CERN SPS.

Figure 3: Hit efficiency vs. bias voltage.

The preliminary analysis of the pulse height distribution for the measurements at CERN yields a mean value of $\sim 14 \mathrm{ke}$. The precise pulse height calibration of the ROC is currently being studied.

\subsection{Results of Detector $2(5 \times 1$ cell ganging $)$}

This device was solely tested at the CERN SPS beam line $\mathrm{H} 6$ with $120 \mathrm{GeV} / \mathrm{c}$ positive charged hadrons. The tracking reconstruction was performed using a high resolution beam telescope, with a spatial resolution of $3 \mu \mathrm{m}$ at the device under test so that the efficiency could be mapped to the spatial coordinates.

The preliminary analysis yields an efficiency of $98.2 \%$ in a contiguous fiducial area. As shown in Figure $\mathbb{A}$, the detector reaches the same efficiency for all tested voltages. The reason of the lower efficiency compared to detector 1 is still under investigation. Most likely this is caused by issues with the bump bonding or an electrostatic issue. The preliminary pulse height in the fiducial region was $\sim 14 \mathrm{ke}$ which is consistent with the result of the first detector. The precise pulse height calibration for the FE-I4B ROC is in the process of being performed.

\section{Conclusion}

We demonstrated a progress in the development of radiation tolerant particle detectors based on pCVD diamond. The preliminary results show two working prototypes of 3D diamond pixel 


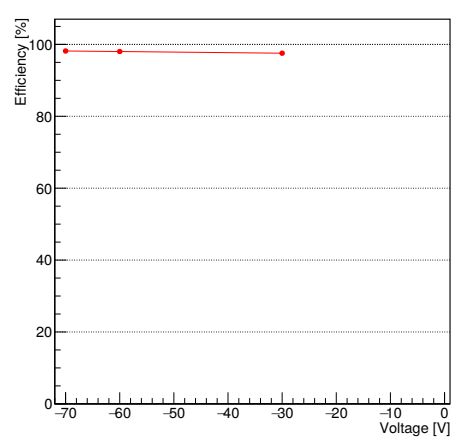

Figure 4: Hit efficiency vs. voltage.

detectors with a cell size of $50 \mu \mathrm{m} \times 50 \mu \mathrm{m}$ and a column diameters of $2.6 \mu \mathrm{m}$. The devices had a total number of $\sim 3500$ cells and the efficiency of the column drilling process is above $99.8 \%$. The first prototypes of small cell 3D diamond pixel detectors read out more charge than any planar pCVD diamond detector. At normal incidence the measured relative hit efficiency of the 3D pixel detectors reached $99.2 \%$ compared to a planar silicon device. In all runs, the tested devices reached efficiencies $>98 \%$.

Future test are planned in order to solve the open questions. Tests at non-normal incidence will hopefully show the origin of the missing $0.8 \%$ in efficiency compared to planar silicon. Further investigations of detector 2 will show whether the slightly lower efficiency is due to the bump bonding or electrostatic issues or something else.

\section{Acknowledgements}

We want to thank Bert Harrop at the Physics Department of the Princeton University as well as Mokhtar Chmeissani, Sebastian Grinstein and David Vazquez Furelos at IFAE for bump bonding the devices. Also our thanks goes to Patrick Salter from the University of Oxford for drilling the 3D column in the diamond sensors. The research leading to these results received funding from the European Union's Horizon 2020 research and innovation program under grant agreement No. 654168. This work was also partially supported by the Swiss National Science Foundation grant \#20FL20_154216, ETH grant 51 15-1, the Swiss Government Excellence Scholarship ESKAS No. 2015.0808, the Royal Society Grant UF120106, the UK Science and Technology Facilities Council Grant ST/P002846/1 and the U.S. Department of Energy through grant DE-SC0010061

\section{References}

[1] D. Contardo, M. Klute, J. Mans, L. Silvestris, and J. Butler, "Technical Proposal for the Phase-II Upgrade of the CMS Detector," Tech. Rep. CERN-LHCC-2015-010. LHCC-P-008. CMS-TDR-15-02, Geneva, Jun 2015.

[2] H. Kagan et al., "Development of Diamond Tracking Detectors for High Luminosity Experiments at the LHC, HL-LHC and Beyond," Tech. Rep. CERN-LHCC-2018-015. LHCC-SR-005, CERN, Geneva, May 2018. 
[3] J. Koike, D. M. Parkin, and T. E. Mitchell, "Displacement threshold energy for type iia diamond," Applied Physics Letters, vol. 60, no. 12, pp. 1450-1452, 1992.

[4] C. D. Clark, P. J. Dean, P. V. Harris, and W. C. Price, "Intrinsic edge absorption in diamond," Proceedings of the Royal Society of London. Series A. Mathematical and Physical Sciences, vol. 277, no. 1370, pp. 312-329, 1964.

[5] H. Feick, "Radiation tolerance of silicon particle detectors for high-energy physics experiments," 1997. Presented on Aug 1997.

[6] J.-W. Tsung et al., "Signal and noise of Diamond Pixel Detectors at High Radiation Fluences.," JINST, vol. 7, p. P09009, Jun 2012.

[7] S. Parker, C. Kenney, and J. Segal, "3D - A proposed new architecture for solid-state radiation detectors," Nuclear Instruments and Methods in Physics Research Section A: Accelerators, Spectrometers, Detectors and Associated Equipment, vol. 395, no. 3, pp. 328 - 343, 1997.

[8] S. M. Pimenov et al., "Femtosecond laser microstructuring in the bulk of diamond," Diamond and Related Materials, vol. 18, no. 2, pp. 196 - 199, 2009.

[9] B. Sun, P. S. Salter, and M. J. Booth, "High conductivity micro-wires in diamond following arbitrary paths," Applied Physics Letters, vol. 105, no. 23, p. 231105, 2014.

[10] A. Kornmayer, T. Müller, and U. Husemann, "Studies on the response behaviour of pixel detector prototypes at high collision rates for the CMS experiment," Nov 2015. Presented 04 Dec 2015.

[11] "Micro/Nano Fabrication Laboratory, Princeton University." https://www.mrfn.org/facilities/princeton-center-complex-materials/ micronano-fabrication-laboratory-mnfl, 2019.

[12] M. Garcia-Sciveres et al., "The FE-I4 Pixel Readout Integrated Circuit," Tech. Rep. ATL-UPGRADE-PROC-2010-001, CERN, Geneva, Jan 2010.

[13] “The Institute for High Energy Physics of Barcelona.” http: / /www . ifae.es/eng/, 2019.

[14] "Institute of Microelectronics of Barcelona IMB-CNM." http://www.imb-cnm.csic.es/index.php/en/, 2019.

[15] "Pion and electron fluxes in piM1." http://aea.web.psi.ch/beam2lines/pim1c.html, 2015.

[16] "CERN SPS H6 Beam Line." http: / / sba.web.cern. ch/sba/BeamsAndAreas/resultbeam. asp?beamline=H6, 2019. 